\title{
Influence of Particle Shape on Bending Rigidity of Colloidal Monolayer Membranes and Particle Deposition during Droplet Evaporation in Confined Geometries
}

\author{
Peter J. Yunker, ${ }^{1}$ Matthew Gratale, ${ }^{1}$ Matthew A. Lohr, ${ }^{1}$ Tim Still, ${ }^{1,2}$ T. C. Lubensky, ${ }^{1}$ and A. G. Yodh $^{1}$ \\ ${ }^{1}$ Department of Physics and Astronomy, University of Pennsylvania, Philadelphia, Pennsylvania 19104, USA \\ ${ }^{2}$ Complex Assemblies of Soft Matter, CNRS-Rhodia-University of Pennsylvania UMI 3254, Bristol, Pennsylvania 19007, USA
}

(Received 27 January 2012; published 1 June 2012)

\begin{abstract}
We investigate the influence of particle shape on the bending rigidity of colloidal monolayer membranes (CMMs) and on evaporative processes associated with these membranes. Aqueous suspensions of colloidal particles are confined between glass plates and allowed to evaporate. Confinement creates ribbonlike air-water interfaces and facilitates measurement and characterization of CMM geometry during drying. Interestingly, interfacial buckling events occur during evaporation. Extension of the description of buckled elastic membranes to our quasi-2D geometry enables the determination of the ratio of CMM bending rigidity to its Young's modulus. Bending rigidity increases with increasing particle anisotropy, and particle deposition during evaporation is strongly affected by membrane elastic properties. During drying, spheres are deposited heterogeneously, but ellipsoids are not. Apparently, increased bending rigidity reduces contact line bending and pinning and induces uniform deposition of ellipsoids. Surprisingly, suspensions of spheres doped with a small number of ellipsoids are also deposited uniformly.
\end{abstract}

PACS numbers: 47.57.J-, 46.70.Hg, 47.55.nb, 47.55.np

When colloidal particles adsorb onto air-water, oil-water, and other such interfaces, novel elastic membranes are created [1,2]. The mechanical properties of these colloidal monolayer membranes (CMMs) can depend on many factors, including surface tension, capillary forces, particle size, shape, hydrophobicity, packing, and interaction potential. The resulting interface phenomenology is rich with physics that influences a wide range of applications from film drying to Pickering emulsion stabilization [3-5]. Nevertheless, full understanding of the elastic character of these membranes remains elusive. Recently, significant progress has been made towards the measurement of the bulk, shear, and Young's moduli of CMMs and towards an understanding of particle-induced interfacial mechanisms $[1,2,6]$. Many effects due to particle shape, for example, can be qualitatively explained by shape-dependent capillary interactions [1,3,7-10]; i.e., stiff membranes induced by ellipsoids at the air-water interface are more difficult to deform $[1,11,12]$. One mechanical property of CMMs that has not as yet been measured is bending rigidity. Bending rigidity is important because the buckling behavior of membranes is controlled by the ratio of bending rigidity $(\kappa)$ to Young's modulus $(E)$ [13], and, as we shall show, the buckling behavior of membranes can substantially affect phenomena such as particle deposition during droplet evaporation. Unfortunately, such measurements are also difficult because constituent particle diameter is often similar to CMM deformation size [14].

In this Letter, we report measurements of the bending rigidity of various colloidal monolayer membranes. We introduce a novel method for extracting bending properties of CMMs which employs evaporating drops in confined geometries and readily permits study of particle-shape effects. To this end, colloidal drops composed of particles with approximately the same chemical composition, but with shapes ranging from spheres to ellipsoids, are confined between two glass plates and left to evaporate [Fig. 1(a)]. During evaporation, the air-water interface is observed to buckle in a manner similar to spherical-shellshaped elastic membranes [15]. To extract membrane bending rigidity, we extend the analytic description of buckled spherical membranes to our quasi-twodimensional geometry [13]. We find that CMM bending rigidity increases with increasing adsorbed-particle shape anisotropy. Besides measurement of bending rigidity, its consequences on particle deposition during evaporation in confined geometries are explored. We discover that increased interfacial bending rigidity dramatically changes particle deposition during evaporation. Spheres can locally pin the three-phase contact line, which then bends around the pinning site and produces an uneven deposition. Conversely, the large bending rigidity induced by adsorbed ellipsoids makes deformation of the contact line energetically costly and ultimately induces uniform deposition. Surprisingly, drops of spheres doped with small numbers of ellipsoids are also deposited relatively uniformly in these confined geometries.

Our experiments utilize micron-sized polystyrene particles with modified shape, stretched asymmetrically to different major-minor diameter aspect ratio, $\alpha$ [16]. The spheres are $1.3 \mu \mathrm{m}$ in diameter; all ellipsoids are stretched from these same $1.3 \mu \mathrm{m}$ spheres. The colloidal drops are confined between two glass slides separated by $38.1 \mu \mathrm{m}$ spacers (Fisher Scientific); qualitatively similar results are 

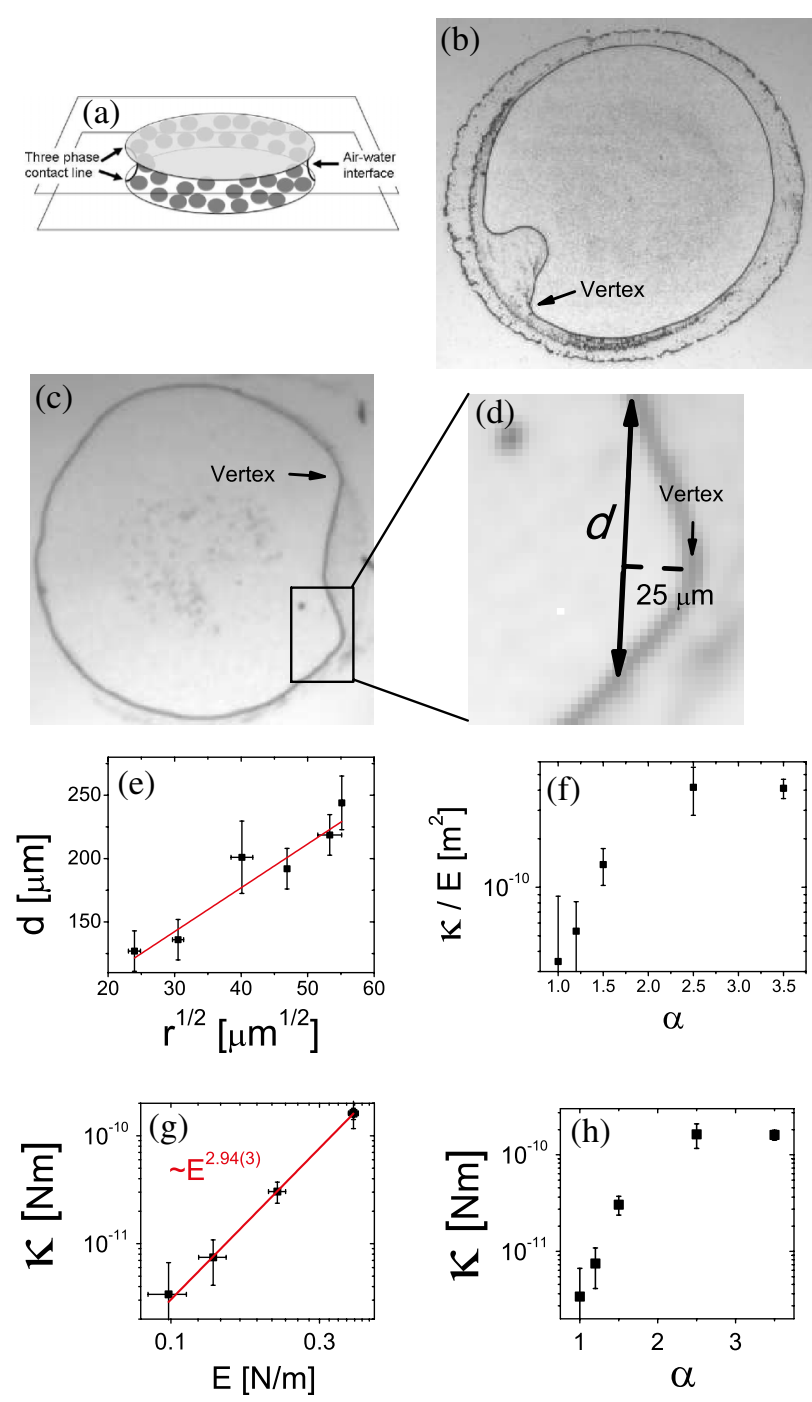

FIG. 1 (color online). (a) Cartoon depicting droplet evaporating in a confined geometry. The particle-populated air-water interface and three-phase contact lines are labeled. (b), (c) Examples of buckling events for confined drops containing anisotropic particles with $\alpha=1.2$ and 1.5 [(b),(c) respectively]. (d) Rim width, $d$ (solid line), is defined here in a magnified image of a buckled region as the interface full-width $25 \mu \mathrm{m}$ from the vertex of the bent air-water interface (see dashed line). (e) $d$ is plotted versus the square root of the drop radius, $r$. (f) Ratio of the bending rigidity, $\kappa$, to the Young's modulus, $E$, is plotted versus $\alpha$. (g) $\kappa$ versus $E$, where $E$ comes from previously reported measurements and calculations [18]. The line represents the best power law fit. (h) $\kappa$ versus $\alpha$.

found for chambers made from slightly hydrophobic cover slips. We investigate the evaporation of these drops, i.e., suspensions containing particles of the same composition but with different major-minor diameter aspect ratios, including spheres $(\alpha=1.0)$, slightly deformed spheres ( $\alpha=1.2,1.5$ ), and ellipsoids ( $\alpha=2.5,3.5$ ). We primarily study the particle volume fraction $\phi=0.01$. (Qualitatively similar results are found for volume fractions ranging from $\phi=10^{-4}$ to 0.05 .) At these low volume fractions, particles densely coat the air-water interface before buckling events occur. The confinement chambers are placed within an optical microscope wherein evaporation is observed at video rates at a variety of different magnifications. Sample temperature is controlled within $0.1{ }^{\circ} \mathrm{C}$.

During evaporation, the air-water interface deforms and crumples [Figs. 1(b) and 1(c)]. The buckling behaviors exhibited by the ribbonlike CMMs in confined geometries are strongly dependent on the shape of the adsorbed particles, and the buckling events appear similar to those observed in spherical-shell elastic membranes [13,17]. Before buckling events occur, particles are maximally packed near the three-phase contact line, regardless of particle shape. Further, because the volume fraction is relatively low, membranes essentially contain a monolayer of particles; i.e., buckling events occur before multilayerparticle membranes form. These buckling events occur in-plane; i.e., the curvature in the $z$ direction does not change after the membrane buckles [18].

To understand this phenomenon, we quantify the elastic properties of the air-water interface with adsorbed particles (i.e., the elastic properties of the CMMs). We first extend analytical descriptions of elastic membranes to our quasi2D geometry wherein observations about bending and buckling geometry are unambiguous [18]. Briefly, the bending energy $\left(\approx \kappa h \zeta^{2} / d^{3}\right.$, where $\kappa$ is the 2D bending rigidity) and stretching energy $\left(\approx E(\zeta / r)^{2} d h\right.$, where $E$ is the 2D Young's modulus) associated with an in-plane buckling event are minimized with respect to the "rim width" of the deformation, $d$ [Figs. 1(b)-1(d)] [13]. Here, $\zeta$ is the radial displacement of the membrane from its initial configuration, $h$ is the chamber height, and $r$ is the in-plane radius of the droplet. More specifically, $d$ is the width of the rim formed by the bent air-water interface, where the deformation bending and stretching energy is concentrated. We measure $d$ as the rim full-width, $25 \mu \mathrm{m}$ in from the rim vertex [defined in Figs. 1(b)-1(d)] [18]. (Note, $d$ is independent of the depth of the invagination. Thus, measurements of $d$ are unaffected by pinning events during buckling.)

This simple approach enables us to extract the ratio of the ribbonlike CMM bending rigidity, $\kappa$, to its Young's modulus, $E$, from measurements of $d$ and $r$ [18]. In particular, minimizing the bending and stretching energy with respect to $d$ yields the relation $\kappa / E=d^{4} /\left(3 r^{2}\right)$. With all other parameters constant, e.g., particle anisotropy, etc., this formula predicts that $d \propto \sqrt{r}$. (Note, this derivation assumes that the interfacial displacement varies little in the $z$ direction; i.e., the air-water interface deflects the same distance at the top, middle, and bottom of the chamber [18].) In Fig. 1(e) we show results from evaporated drops of particles with anisotropy $\alpha=1.2$ and with different initial values of $r$, plotting $d$ versus $\sqrt{r}$. A good linear relationship is observed (coefficient of determination, $R^{2}=0.93$ ), implying 
that our analysis is self-consistent. Similar linear results were found for other values of $\alpha$.

We thus extract and plot $\kappa / E$ for evaporating drops of particles with different $\alpha$ [Fig. 1(f)]. Notice, $\kappa / E$ increases with increasing $\alpha$, implying that as $\alpha$ increases, $\kappa$ increases faster than $E$; i.e., $\kappa / E$ is larger for ellipsoids $(\alpha=2.5$ and 3.5) than for spheres $(\alpha=1.0)$. CMM Young's modulus is known to increase with $\alpha[1,2,6]$. Utilizing previously reported measurements and calculations of $E$ ([1,6]; see [18] for a list of these numbers), we plot $\kappa$ versus $E$ [Fig. $1(\mathrm{~g})]$ and find that $\kappa \propto E^{2.94(3)}$. This observation is consistent with theoretical models which predict $\kappa \propto E^{3}$ [13]; however, the full physical origin of this connection is unclear. (Note, while $\kappa \propto E^{3}$ and $\kappa / E=$ $d^{4} /\left(3 r^{2}\right)$ may appear contradictory, they are consistent and imply that $d \propto E^{1 / 2}$. This relationship is supported by our experimental data [18].) Finally, we use previously reported measurements and calculations of $E$ to isolate and estimate the ribbonlike CMM bending rigidity [Fig. 1(h)]. Clearly, membrane bending becomes more difficult with increasing particle anisotropy.

We next turn our attention to the consequences of increased bending rigidity on evaporation processes in confined geometries, specifically particle deposition during drying. Substantial effort has now yielded an understanding of the so-called coffee ring effect and some ability to control particle deposition from sessile drops [5,19]. However, much less is known about particle deposition in confined geometries, despite the fact that many real systems [20] and applications [21] feature evaporation in geometries wherein the air-water interface is present only at the system edges. Recent experiments have explored evaporation of confined drops containing spheres [15,22], and their behaviors differ dramatically from sessile drops containing spheres. In the confined case, as noted previously, particles are pushed to the ribbonlike air-fluid interface, and, as evaporation proceeds, the particle-covered air-water interface often undergoes the buckling events described above.

We find that suspended particle shape produces dramatically different depositions as a result of the varying CMM bending moduli. In Figs. 2(a)-2(e), the final deposition of particles is shown for $\alpha=1.0,1.2,1.5,2.5,3.5$, respectively. Spheres and slightly stretched spheres are deposited heterogeneously, and anisotropic ellipsoids are distributed relatively more uniformly. To describe the final deposition of particles more quantitatively, we plot the fraction of initial droplet area covered by deposited particles after drying, $f$ (as introduced in [23]), as a function of anisotropy $\alpha$ [Fig. 2(f)] [18]. Note, for uniformly deposited particles, the area fraction (based on the initial volume fraction, initial volume, chamber height, and particle size) would be $\sim 0.4$; thus, regions with area fraction $\approx 0.4$ are considered as covered. The fraction of the area covered with particles is observed to increase with $\alpha$. For $\alpha=1.2$ and 1.5, $f$ increases modestly. For $\alpha=2.5$, the
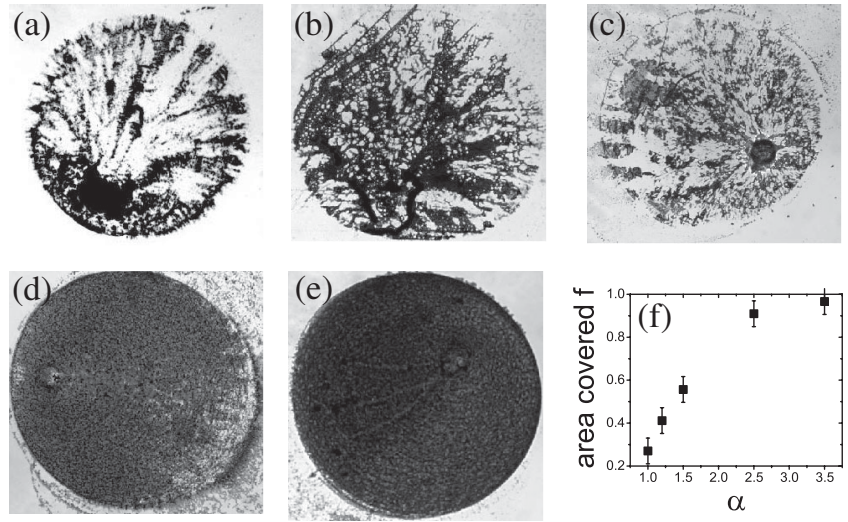

FIG. 2. Image of the final deposition of particles with majorminor diameter aspect ratio $\alpha=1.0,1.2,1.5,2.5,3.5$ [(a)-(e), respectively]. (f) The area fraction covered by particles after evaporation is complete, $f$, for suspensions of particles as a function of their aspect ratio $\alpha$.

deposition is very uniform, and for $\alpha=3.5$, virtually the entire area is covered uniformly.

High magnification images reveal why spheres and slightly stretched particles deposit unevenly, while ellipsoids deposit more uniformly [Figs. 3(a)-3(d)]. Spheres and slightly stretched spheres often pin the air-water interface, preventing its motion. In fact, spheres can pin the air-water interface even in very dilute suspensions, i.e., $\phi<10^{-4}$. As evaporation continues, the CMM interface bends around the pinning site [Fig. 3(a)]. Then, it either pinches off, leaving particles behind, or it remains connected to the pinned site, leading to water flow into the narrow channel that has formed; the latter flow carries particles towards the pinning site [Fig. 3(a) and 3(b)] producing "streaks" of deposited particles [Fig. 3(c)]. Temporal and spatial heterogeneities along the interface due to these described effects lead to heterogeneous deposition of spherical particles during evaporation.

When ellipsoids approach the drop edge they also adsorb onto the air-water interface forming ribbonlike CMMs [Fig. 3(d)] [1,3,8,9]. However, the ellipsoids induce substantial capillary deformations on the air-water interface, creating an elastic membrane with a high bending rigidity. Ellipsoids can also pin the contact line, but bending of the CMM interface around a pinned contact line requires an energetically costly rearrangement of ellipsoids aggregated on the CMM; i.e., attractive particle-particle capillary interactions must be overcome (even at very small $\phi$ ). Conversely, bending of the contact line costs little energy to spheres on the interface because sphere-sphere capillary interactions on the interface are much weaker than for ellipsoids [8,24]. As evaporation continues, the ellipsoidCMM contact line recedes radially, and the ellipsoids near the contact line are deposited on the substrate. This behavior is similar to convective assembly techniques wherein a drying front is created by pulling the substrate away from 


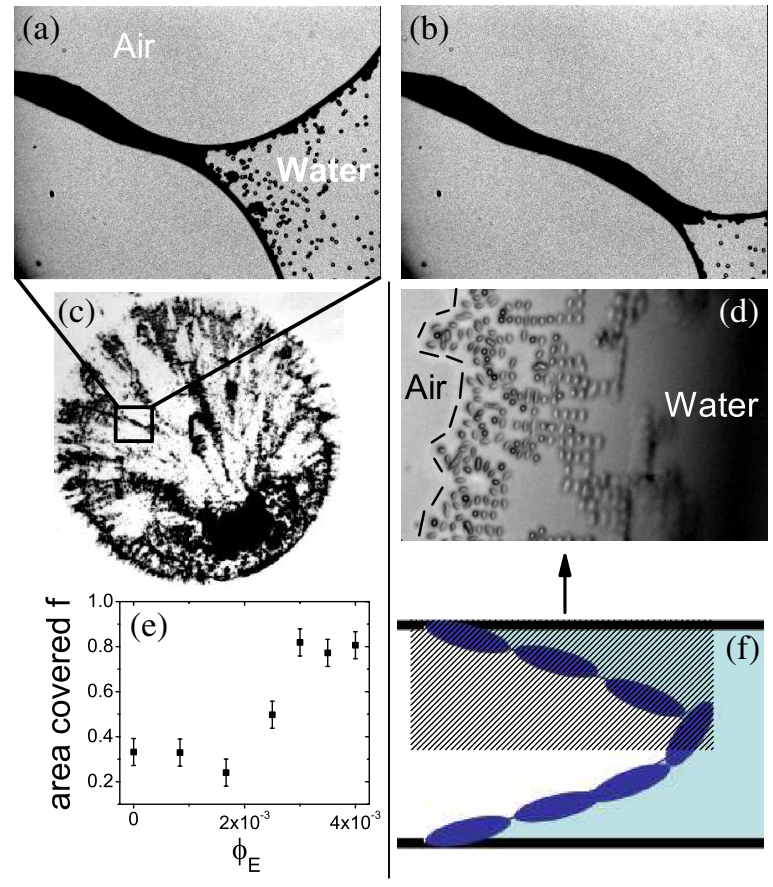

FIG. 3 (color online). (a) Image of a pinned region of the airwater interface $(\alpha=1.0)$. When the pinned section does not "snap" off, it leaves behind a channel. (b) At a later time $[\sim 100 \mathrm{~s}$ after (c)], the channel extends, and more particles flow into it, producing a very heterogeneous deposition. (c) Image of the final deposition of particles with major-minor diameter aspect ratio $\alpha=1$. 0 . The box indicates the deposit left behind by the event depicted in (a) and (b). (d) Image of a colloidal monolayer near the three-phase contact line in a drop containing ellipsoids $(\alpha=3.5)$. The three-phase contact line is labeled with a dashed line on the left side of the image. Particles are adsorbed on the air-water interface, forming a monolayer, as evidenced by the fact that particles become more out of focus from left to right as the air-water interface curves. A cartoon (f) shows a side view of the experimental image. (e) The fraction of area covered by particles, $f$, for suspensions of $200 \mathrm{~nm}$ diameter spheres doped with different amounts of ellipsoids, represented by the ellipsoid volume fraction, $\phi_{E}$

the contact line or by heating a confined drop near the contact line; in each case a thin film is thus formed that leads to the creation of a monolayer (e.g., [25]). The present system, by contrast, has neither moving nor mechanical parts. Uniform coatings are created essentially as a result of shape-induced capillary attractions which produce CMMs that are hard to bend.

To further elucidate the effects of particle shape on deposition, suspensions of $200 \mathrm{~nm}$ spheres $(\alpha=1.0)$ with $\phi=0.02$ were combined with suspensions containing micron-sized ellipsoids $(\alpha=3.5)$ at lower volume fractions, $\phi=0$ to $4.0 \times 10^{-3}$. The resulting colloidal drops were evaporated in the same confined geometries. The addition of a very small number of ellipsoids has no effect on the deposition of spheres $\left(\phi \leq 1.7 \times 10^{-3}\right)$. Surprisingly, the addition of a larger, but still small, number of ellipsoids leads to a uniform deposition of both ellipsoids and spheres, i.e., $f \approx 0.8$, despite the fact that spheres outnumber ellipsoids by a significant factor $\left(10^{3}-10^{4}\right)$ [Fig. 3(e)]. Apparently, spheres do not prevent ellipsoids from adsorbing on the air-water interface, and the CMM bending rigidity is dominated by the presence of ellipsoids. Thus, the membrane still resists bending around pinning sites. This behavior in confined geometries is different than that of sessile drops wherein it was discovered that if the spheres are larger than the ellipsoids, then the spheres are distributed uniformly after drying, but if the spheres are smaller than the ellipsoids, then they exhibit the coffee ring effect [5]. From this perspective, it is somewhat surprising that small spheres are deposited uniformly from droplets doped with small numbers of ellipsoids and confined between glass plates.

Again, the high bending modulus produced by ellipsoids on the CMM helps explain the observations. Both spheres and ellipsoids attach to the air-water interface. Ellipsoids deform the air-water interface, creating an effective elastic membrane with a high bending rigidity. When enough ellipsoids are present, pinning and bending the interface becomes energetically costly and the spheres (and ellipsoids) are deposited as the interface recedes.

To summarize, ellipsoids adsorbed on the air-water interface create an effective elastic membrane, and, as particle anisotropy aspect ratio increases, the membrane's bending rigidity increases faster than its Young's modulus. As a result, when a drop of a colloidal suspension evaporates in a confined geometry, the different elastic properties produce particle depositions that are highly dependent on particle shape. This observed increase in bending rigidity with particle shape aspect ratio holds important consequences for applications of colloidal monolayer membranes as well. For example, increased bending rigidity may help stabilize interfaces (e.g., Pickering emulsions [3]) and thus could be important for many industrial applications, e.g., food processing $[4,26]$. In a different vein, our observations suggest that CMMs in confined geometries may be a convenient model system to study buckling processes that are relevant for other systems, e.g., polymeric membranes [27], biological membranes [28], and nanoparticle membranes [29].

We thank Erin M. Buckley, Piotr Habdas and Kevin B. Aptowicz for helpful discussions, and we gratefully acknowledge financial support from the National Science Foundation through DMR-0804881, the PENN MRSEC DMR11-20901, and from NASA NNX08AO0G. T.S. acknowledges support from DAAD.

[1] B. Madivala, J. Fransaer, and J. Vermant, Langmuir 25, 2718 (2009).

[2] L. M. C. Sagis, Soft Matter 7, 7727 (2011); P. Moldenaers and J. Vermant, Phys. Chem. Chem. Phys. 9, 6463 (2007). 
[3] B. Madivala, S. Vandebril, J. Fransaer, and J. Vermant, Soft Matter 5, 1717 (2009).

[4] J. Vermant, Nature (London) 476, 286 (2011).

[5] P. J. Yunker, T. Still, M. A. Lohr, and A. G. Yodh, Nature (London) 476, 308 (2011).

[6] M. G. Basavaraj, G. G. Fuller, J. Fransaer, and J. Vermant, Langmuir 22, 6605 (2006); D. Vella, P. Aussillous, and L. Mahadevan, Europhys. Lett. 68, 212 (2004).

[7] D. Stamou, C. Duschl, and D. Johannsmann, Phys. Rev. E 62, 5263 (2000).

[8] J. C. Loudet, A. G. Yodh, and B. Pouligny, Phys. Rev. Lett. 97, 018304 (2006); J. C. Loudet, A. M. Alsayed, J. Zhang, and A. G. Yodh, Phys. Rev. Lett. 94, 018301 (2005).

[9] N. Bowden, F. Arias, T. Deng, and G. M. Whitesides, Langmuir 17, 1757 (2001); A. B. D. Brown, C. G. Smith, and A. R. Rennie, Phys. Rev. E 62, 951 (2000); S. D. J. Guzowski, M. Tasinkevych, and S. Dietrich, Phys. Rev. E 84, 031401 (2011).

[10] M. Mittal and E. M. Furst, Adv. Funct. Mater. 19, 3271 (2009).

[11] N. Tsapis, E. R. Dufresne, S.S. Sinha, C.S. Riera, J. W. Hutchinson, L. Mahadevan, and D. A. Weitz, Phys. Rev. Lett. 94, 018302 (2005); L. Pauchard and Y. Couder, Europhys. Lett. 66, 667 (2004).

[12] L. Botto, L. Yao, R. L. Leheny, and K. J. Stebe, Soft Matter 8, 4971 (2012).

[13] L. D. Landau, L. P. Pitaevskii, E. M. Lifshitz, and A. M. Kosevich, Theory of Elasticity (Butterworth-Heinemann, Oxford, England, 1986), 3rd ed., Vol. 7.

[14] K. D. Danov, P. A. Kralchevsky, and S. D. Stoyanov, Langmuir 26, 143 (2010); R. Aveyard, J. H. Clint, and T. S. Horozov, Phys. Chem. Chem. Phys. 5, 2398 (2003); H. Schwartz, Y. Harel, and S. Efrima, Langmuir 17, 3884 (2001); C. Planchette, E. Lorenceau, and A. Biance, Soft Matter 8, 2444 (2012).

[15] J. Leng, Phys. Rev. E 82, 021405 (2010); L. Pauchard, M. Mermet-Guyennet, and F. Giorgiutti-Dauphine, Chemical Engineering and Processing 50, 483 (2011); L. Daubersies and J. B. Salmon, Phys. Rev. E 84, 031406 (2011).

[16] B. Felder, Helv. Chim. Acta 49, 440 (1966); J. A. Champion, Y. K. Katare, and S. Mitragotri, Proc. Natl. Acad. Sci. U.S.A. 104, 11901 (2007); C. C. Ho, A. Keller, J. A. Odell, and R. H. Ottewill, Colloid Polym. Sci. 271, 469 (1993).
[17] G. A. Vliegenthart and G. Gompper, New J. Phys. 13, 045020 (2011).

[18] See Supplemental Material at http://link.aps.org/ supplemental/10.1103/PhysRevLett.108.228303 for a discussion of additional experimental and theoretical details.

[19] R. D. Deegan, O. Bakajin, T. F. Dupont, G. Huber, S. R. Nagel, and T.A. Witten, Nature (London) 389, 827 (1997); N.D. Denkov, O.D. Velev, P.A. Kralchevsky, I. B. Ivanov, H. Yoshimura, and K. Nagayama, Nature (London) 361, 26 (1993); H. Hu and R. G. Larson, J. Phys. Chem. B 106, 1334 (2002); T.P. Bigioni, X.-M. Lin, T. T. Nguyen, E. I. Corwin, T. A. Witten, and H. M. Jaeger, Nature Mater. 5, 265 (2006); H. Hu and R. G. Larson, J. Phys. Chem. B 110, 7090 (2006); B. M. Weon and J. H. Je, Phys. Rev. E 82, 015305 (2010); T. Kajiya, W. Kobayashi, T. Okuzono, and M. Doi, J. Phys. Chem. B 113, 15460 (2009); V. X. Nguyen and K. J. Stebe, Phys. Rev. Lett. 88, 164501 (2002); B. J. Park and E. M. Furst, Soft Matter 6, 485 (2010); H. B. Eral, D. M. Augustine, M. H. G. Duits, and F. Mugele, Soft Matter 7, 4954 (2011); A. P. Sommer and N. Rozlosnik, Cryst. Growth Des. 5, 551 (2005).

[20] P. Faure and P. Coussot, Phys. Rev. E 82, 036303 (2010); F. Clement and J. Leng, Langmuir 20, 6538 (2004); M. Scheel, R. Seemann, M. Brinkmann, M. Di Michiel, A. Sheppard, B. Breidenbach, and S. Herminghaus, Nature Mater. 7, 189 (2008).

[21] E. Berthier, J. Warrick, H. Yu, and D. J. Beebe, Lab Chip 8, 852 (2008); C. Cottin, H. Bodiguel, and A. Colin, Phys. Rev. E 84, 026311 (2011).

[22] S. H. Kang, N. Wu, A. Grinthal, and J. Aizenberg, Phys. Rev. Lett. 107, 177802 (2011).

[23] R. D. Deegan, Phys. Rev. E 61, 475 (2000).

[24] B. J. Park and E. M. Furst, Soft Matter 7, 7676 (2011).

[25] P. Kumnorkaew, Y.-K. Ee, N. Tansu, and J. F. Gilchrist, Langmuir 24, 12150 (2008); M. Mittal and E. M. Furst, Adv. Funct. Mater. 19, 3271 (2009).

[26] E. Dickinson, Curr. Opin. Colloid Interface Sci. 15, 40 (2010).

[27] M. Ulbricht, Polymer 47, 2217 (2006).

[28] D. A. Fletcher and R. D. Mullins, Nature (London) 463, 485 (2010).

[29] K.E. Mueggenburg, X.-M. Lin, R.H. Goldsmith, and H. M. Jaeger, Nature Mater. 6, 656 (2007). 